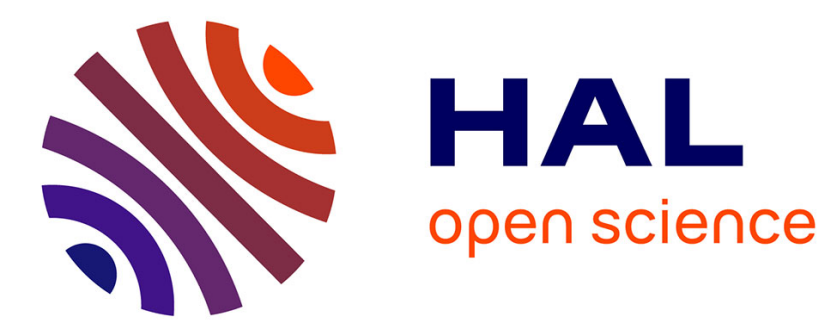

\title{
Comparison of Mixed-Integer Linear Models for Fuel-Optimal Air Conflict Resolution With Recovery
}

Jérémy Omer

\section{To cite this version:}

Jérémy Omer. Comparison of Mixed-Integer Linear Models for Fuel-Optimal Air Conflict Resolution With Recovery. IEEE Transactions on Intelligent Transportation Systems, 2015, 16 (6), pp.3126-3137. 10.1109/TITS.2015.2434942 . hal-02099641

\section{HAL Id: hal-02099641 https://hal.science/hal-02099641}

Submitted on 15 Apr 2019

HAL is a multi-disciplinary open access archive for the deposit and dissemination of scientific research documents, whether they are published or not. The documents may come from teaching and research institutions in France or abroad, or from public or private research centers.
L'archive ouverte pluridisciplinaire HAL, est destinée au dépôt et à la diffusion de documents scientifiques de niveau recherche, publiés ou non, émanant des établissements d'enseignement et de recherche français ou étrangers, des laboratoires publics ou privés. 


\title{
Comparison of Mixed Integer Linear Models for Fuel-Optimal Air Conflict Resolution with Recovery
}

\author{
Jérémy Omer
}

\begin{abstract}
Any significant increase in current levels of air traffic will need the support of efficient decision-aid tools. One of the tasks of air traffic management is to modify trajectories when necessary to maintain a sufficient separation between pairs of aircraft. Several algorithms have been developed to solve this problem, but the diversity in the underlying assumptions makes it difficult to compare their performance. In this article, separation is maintained through changes of heading and velocity while minimizing a combination of fuel consumption and delay. For realistic trajectories, the speed is continuous with respect to time, the acceleration and turning rate are bounded, and the planned trajectories are recovered after the maneuvers. After describing the major modifications to existing models that are necessary to satisfy this definition of the problem, we compare three mixed integer linear programs. The first model is based on a discretization of the airspace, and the second relies on a discretization of the time horizon. The third model implements a time decomposition of the problem; it allows only one initial maneuver, and it is solved periodically with a receding horizon to build a complete trajectory. The computational tests are conducted on a benchmark of artificial instances specifically built to include complex situations. Our analysis of the results highlights the strengths and limits of each model. The time decomposition proves to be an excellent compromise.
\end{abstract}

Index Terms-Air traffic control, Conflict resolution, Mixed integer linear programming.

\section{INTRODUCTION}

The airspace is a crowded environment, so air traffic management (ATM) is necessary for both security and economic reasons. ATM is organized into successive layers corresponding to levels of anticipation that converge toward real-time. The last layer is air traffic control (ATC); for portions of the airspace outside the direct vicinity of airports, it involves monitoring traffic, establishing communication with pilots, and taking actions to ensure the fluidity and safety of the traffic.

For safety, ICAO [1] defines required separation distances that depend on airspace class. Two aircraft are considered separated if they respect either a required horizontal or vertical distance, else the aircraft are in conflict. A potential conflict occurs when the predicted trajectories of two aircraft are in

Jérémy Omer is with the École Polytechnique de Montréal, 2900 bd. Edouard-Montpetit, Montreal (QC), Canada H3T 1J4 and the Group for Research in Decision Analysis, HEC Montréal, 3000 ch. de la Cte-SainteCatherine Montreal (QC), Canada H3T 2A7; e-mail: jeremy.omer@gerad.ca

The research presented in this article was funded by ONERA, the French Aerospace Lab, Toulouse, France.

Digital Object Identifier 10.1109/TITS.2015.2434942

2015 IEEE. Personal use of this material is permitted. Permission from IEEE must be obtained for all other uses, in any current or future media including reprinting/republishing this material for advertising or promotional purposes, creating new collective works, for resale or redistribution to servers or lists, or reuse of any copyrighted component of this work in other works. conflict at some future time. The ATC operator must detect potential conflicts, determine maneuvers that lead to conflictfree trajectories, and communicate the maneuvers to pilots. The second task is usually referred to as conflict resolution (CR). Automated CR could benefit the overall ATM. Indeed, the inclusion of an automated CR in the toolbox available to controllers could lead to a major increase in airspace capacity [2]. Since the CR problem is also intrinsically difficult, it has been the focus of a large literature. The review articles written by Kuchar and Yang [3] and Martín-Campo [4] allow to understand, compare and contextualize the large diversity of existing studies. These surveys also show that an ideal approach should rely on general assumptions, fit the nature of the problem, and be computationally efficient. In practice, $\mathrm{CR}$ algorithms represent a compromise among these three aspects.

Optimal control methods search for a continuous-time control law optimizing a cost functional. Since the aircraft trajectories involve continuous-time positions, speeds, and accelerations, optimal control is a logical candidate for solving the CR problem. Complete models are derived in [5]-[7]. The optimal solution can be found for simple cases with two aircraft and a constant velocity [5]. However, numerical methods are necessary to solve the problem under more general assumptions.

If the aircraft fly at the same altitude, one approach is to limit the possible maneuvers to a finite set of heading and/or speed changes. With this restriction, Bicchi and Pallottino [6] suggest a heuristic that builds conflict-free trajectories with sequences of straight lines and circular arcs. Frese and Beyerer [8] focus on instantaneous heading changes with constant speed and velocity changes with constant heading to solve the problem through a tree-exploration technique. With the same maneuvers, Durand et al. [9] develop a genetic algorithm to handle complex situations.

A second approach is to formulate the CR problem with discrete time. For instance, Raghunathan et al. [7] sample the time interval to focus on a finite set of variables and constraints. The resulting model is a nonlinear program (NLP) with nonconvex separation constraints. The NLP can be solved numerically, but fast algorithms cannot guarantee more than a local optimum. Moreover, when solving nonconvex NLPs, these algorithms are sensitive to the point from which they are started. Borrelli et al. [10] show that this results in large computational times and oscillations in the costs of the solutions.

For faster solution and guaranteed convergence, the nonlinear constraints and objective function of the NLP can be approximated with linear equations involving integer variables 
to obtain a mixed integer linear program (MILP). MILPs have been very popular in ATC, because they may achieve a good approximation of the NLP (see [11]), and implementations of state-of-the-art algorithms (e.g., $\mathrm{CPLEX}^{1}$ or Gurobi [12]) find optimal solutions of large instances in a reasonable time. For instance, time-discretized MILPs are developed in [13]-[16].

An extreme time sampling is to take only the two extremities of the time interval. In this case, the conflicts resolution must be achieved with one initial maneuver per aircraft, thus leading to a simpler form of the NLP. In particular, the separation constraints become a disjunction of two linear constraints (see [17]-[20]).

MILPs also arise from space discretization. Instead of sampling the time interval, these models reduce the airspace to a finite set of important points, namely the initial and final points of the trajectories and the points where pairs of trajectories intersect. Since space discretization makes it hard to represent geographic deviations from the predicted trajectories, in these models the maneuvers are usually restricted to speed changes [21]-[24]. By introducing lateral shifts similar to those described in [19], Omer [25] develops a space-discretized model that also includes heading changes.

Despite the abundant literature on $\mathrm{CR}$, there has been little experimental comparison of different models. Frese and Beyerer [26] conduct an experimental study for the avoidance of collisions between cars. In [10], [16], results are presented for an NLP and an MILP and [16] shows that the MILP may be used as a first step toward the final result by providing a starting point for the NLP [16]. The first motivation for this article is to add to the body of knowledge by conducting an experimental comparison of CR models. Since a large proportion of the existing models are MILPs and they represent a wide range of options, this work focuses on MILPs. Based on the literature review above, we compare three different families of MILPs: time-discretized, one-maneuver, and space-discretized models.

In this work we consider the restriction of the $\mathrm{CR}$ problem to deterministic and planar motion, so the main characteristics of the problem are the following:

- fuel consumption and delays are minimized;

- speed and heading changes are allowed;

- speed and heading are continuous functions of time;

- velocity, acceleration, and turning rate are bounded;

- aircraft must revert to their planned trajectories.

For a meaningful comparison of the three families of MILPs, the compared models must all be based on the same requirements. Our first major contribution is thus to modify existing time-discretized and one-maneuver models with this goal in mind. Our second major contribution is an experimental comparison of the three models. We generate a large set of benchmark instances to ensure that the conclusions illustrate general tendencies, and we highlight the main features of each family of model. The most interesting result is that the onemaneuver model emerges as an excellent compromise.

Our approach is based on the formal definition of the

${ }^{1}$ CPLEX is freely available for academic and research purposes under the IBM academic initiative: http://www-03.ibm.com/ibm/university/academic problem given in Section II. In Section III, we briefly describe a previously developed space-discretized model. Our original modeling contribution includes the insertion of fuel consumption in a time-discretized model in Section IV and a larger revision of a one-maneuver model in Section V. Based on experimental tests, we compare the models in Section VI.

\section{FORMAL DEFINITION OF THE CONFLICT RESOLUTION PROBLEM}

The CR problem aims to keep a set $\mathcal{A}$ of aircraft separated on a time interval $[0, T]$. Denoting $\mathcal{C}$ the set of pairs of distinct aircraft in $\mathcal{A}$, a new trajectory is planned for each aircraft in $\mathcal{A}$ so that each pair in $\mathcal{C}$ respects the required separation distances on $[0, T]$. The control horizon $T$ may be related to the transit time through control sectors, so the value of $T$ typically ranges from 10 to 15 minutes.

In this section we define the specific CR problem studied in the rest of the paper. The definition covers aircraft dynamics, separation constraints, recovery of the planned trajectories and cost minimization.

\section{A. Dynamics of the aircraft}

The motion of an aircraft $A_{i} \in \mathcal{A}$ is described by its position $\boldsymbol{p}_{i}(t)$, speed $\boldsymbol{v}_{i}(t)$, and acceleration $\boldsymbol{u}_{i}(t)$ at each time $t \in$ $[0, T]$. The particular problem we are focusing on deals with planar motion, which allows us to capture a large part of the conflicts between cruising aircraft. Stated otherwise, $\boldsymbol{p}_{i}(t)$, $\boldsymbol{v}_{i}(t)$, and $\boldsymbol{u}_{i}(t)$ are assumed to be two-dimensional vectors, so separation is achieved through heading and speed maneuvers. Moreover, we restrict the problem to deterministic motion. The studies by Erzberger and Paielli [27], and Chaloulos and Lygeros [28] justify that this restriction should not have major consequences in the conclusions of this study. Indeed, [27], [28] show that the errors in trajectory prediction do not exceed a few nautical miles for the considered control horizon. In Section VII, we discuss how uncertainties could be taken into account in a future comparative study.

As is standard for the CR problem, we model the motion as a double integrator:

$$
\left(\begin{array}{c}
\frac{\mathrm{d} \boldsymbol{p}_{i}(t)}{\mathrm{d} t} \\
\frac{\mathrm{d} \boldsymbol{v}_{i}(t)}{\mathrm{d} t}
\end{array}\right)=\left(\begin{array}{c}
\boldsymbol{v}_{i}(t) \\
\boldsymbol{u}_{i}(t)
\end{array}\right) .
$$

A reasonable level of realism is ensured by assuming that acceleration is stepwise constant. In other words, the maneuvers are executed with a constant acceleration vector, and the speed vector remains constant between two consecutive maneuvers. This assumption is consistent with the continuity of speed with respect to time and the need to execute maneuvers smoothly.

The performance of an aircraft imposes a minimum and a maximum speed, $V^{\min }$ and $V^{\max }$. Moreover, as specified in [29], large velocity decreases below the preferred value are usually not appreciated by pilots. As a consequence, the minimum velocity is the larger of the value based on the aircraft's performance and that based on the pilots' preferences. In addition, the comfort of the passengers leads to maximum values, $U^{\max }$ and $\omega^{\max }$, for the derivatives of velocity and 
heading, which are called acceleration and yaw rate in what follows. These constraints are formalized as: $\forall t \in[0, T]$,

$$
\begin{gathered}
V_{i}^{\min } \leq V_{i}(t) \leq V_{i}^{\max } \\
\left|\frac{\mathrm{d} \chi_{i}(t)}{\mathrm{d} t}\right| \leq \omega_{i}^{\max } \text { and }\left|\frac{\mathrm{d} V_{i}(t)}{\mathrm{d} t}\right| \leq U_{i}^{\max },
\end{gathered}
$$

where $\chi_{i}(t)$ is the heading and $V_{i}(t)$ is the velocity of $A_{i}$ at time $t$.

\section{B. Ensuring separation}

Since the modeled aircraft dynamics are restricted to the horizontal plan, only the horizontal separation $D$ is considered. The separation constraints are thus formulated as:

$$
\left\|\boldsymbol{p}_{j}(t)-\boldsymbol{p}_{i}(t)\right\| \geq D, \forall\left(A_{i}, A_{j}\right) \in \mathcal{C}, \forall t \in[0, T],
$$

where $\|\cdot\|$ is the Euclidean norm.

\section{Reverting to the planned trajectory}

Trajectory recovery reflects the natural idea that CR should minimize perturbations to the overall trajectory of an aircraft. Moreover, several projects consider a trajectory-based ATM in which both the airlines and ATC commit to do all they can to keep each aircraft as close as possible to a previously negotiated business trajectory (BT) (see [30]).

In this article, both space and time deviations from the BT are controlled. A constraint is then added for each aircraft to recover the course of its planned trajectory. For this, the final position must be on the straight line directed by the planned final speed and going through the planned final position:

$$
\left(\boldsymbol{p}_{i}(T)-\boldsymbol{p}_{i}^{T}\right) \otimes \boldsymbol{v}_{i}^{T}=0, \forall A_{i} \in \mathcal{A},
$$

where $\boldsymbol{p}_{i}^{T}$ and $\boldsymbol{v}_{i}^{T}$ are the planned position and speed of $A_{i}$ at time $T$ and $\otimes$ is the vector product. The temporal aspect of trajectory recovery is taken into consideration through a penalty in the cost function rather than a hard constraint. This issue is dealt with in the next section.

\section{Cost minimization}

The economic efficiency of a flight is usually measured by duration and fuel consumption. Fuel-optimal conflict-free trajectories are thus determined, for instance, in [18]. In this article, the trajectories minimize a combination of the fuel consumption and the time deviations from the BT at time $T$.

To derive fuel consumption we use the physical model described in the BADA user manual [31]. Sample consumption profiles are plotted in Figure 1.

To penalize the time deviation, we estimate the cost of a delay as the fuel cost that would be incurred to make up with the delay created by the maneuvers. Since this operation is not an emergency, the speed should not be pushed to its limit. Denoting $V_{i}^{\text {nom }}$ the nominal speed of $A_{i}, \frac{1}{2}\left(V_{i}^{\text {nom }}+V_{i}^{\max }\right)$ or $\frac{1}{2}\left(V_{i}^{\text {nom }}+V_{i}^{\text {min }}\right)$ are acceptable speeds to make up with a positive or negative delay. The penalties for being behind or ahead of the BT, $\rho_{i}^{-}$and $\rho_{i}^{+}$, are thus computed as the additional fuel consumption needed to return to the BT. A detailed description of the computation of $\rho_{i}^{-}$and $\rho_{i}^{+}$is given in [11].

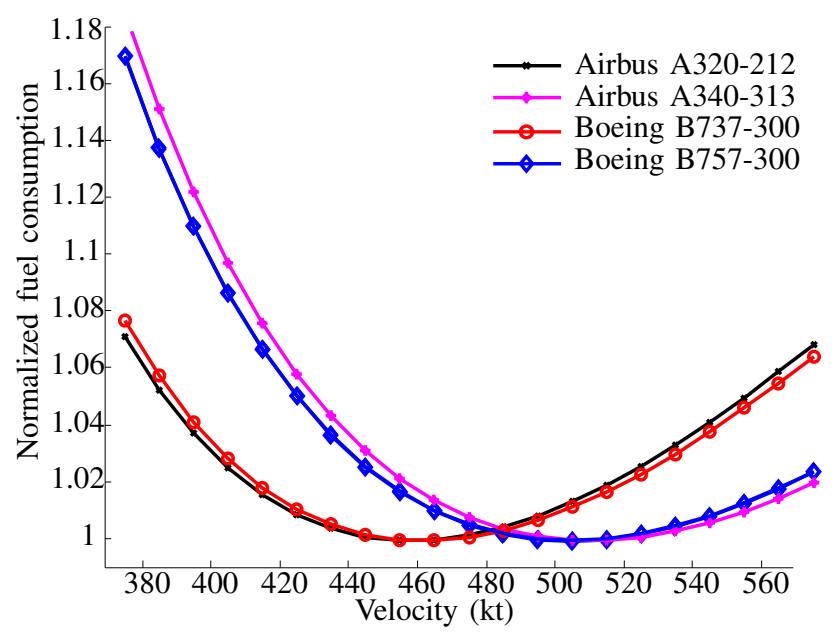

Fig. 1: Fuel consumption per distance unit; the profiles are normalized by setting the minimum consumption equal to 1.0

\section{SPACE-DISCRETIZED MODEL}

Space discretization focuses on the points of the airspace that are most likely to be important for the conflict resolution. These are the measured positions of the aircraft at $t=0$, the predicted final positions of the aircraft, and the conflict points, i.e., the points where the trajectories of two aircraft intersect. Although it is not identified as such, a space discretization is used in [21], [22], [24] to derive a model involving only speed maneuvers. The main contribution of a previous work [25] is to include heading maneuvers. Since we implement the same model, we describe only the principles of space discretization (Section III-A) and the assumptions made to allow for a linear formulation with heading maneuvers (Section III-B). The complete mathematical formulation can be found in the original description of the model.

\section{A. Principle of the space discretization}

For given traffic, space discretization leads to a directed graph $(\mathcal{N}, \mathcal{E})$, where $\mathcal{N}$ is the union of the following set of nodes:

- $\mathcal{N}_{I}$ : nodes corresponding to the initial positions;

- $\mathcal{N}_{C}$ : nodes corresponding to the conflict points;

- $\mathcal{N}_{T}$ : nodes corresponding to the positions at time $T$.

Set $\mathcal{E}$ is then built by adding, for each aircraft, the pairs of consecutive nodes over which the aircraft flies. The conflict graph is completely built based on the BTs. In the model, the structure of the graph is thus fixed. The variable features of the graph are the characteristics of the edges. For instance, a speed change modifies the flight time, and heading maneuvers stretch out the length of an edge while introducing a lateral shift with respect to the BT. Figure 2 illustrates the structure of a conflict graph for a situation involving three aircraft: $n_{1}$ and $n_{3}$ are the intial and final nodes of $A_{1}$ and $n_{2}$ is a conflict node; $\left(A_{1}, n_{1}, n_{2}\right)$ and $\left(A_{1}, n_{2}, n_{3}\right)$ are the edges corresponding to the BT of $A_{1}$.

In Section II, the separation constraints focus on the positions of two aircraft at each time, whereas in a spatial discretization they focus on the times the two aircraft fly over 


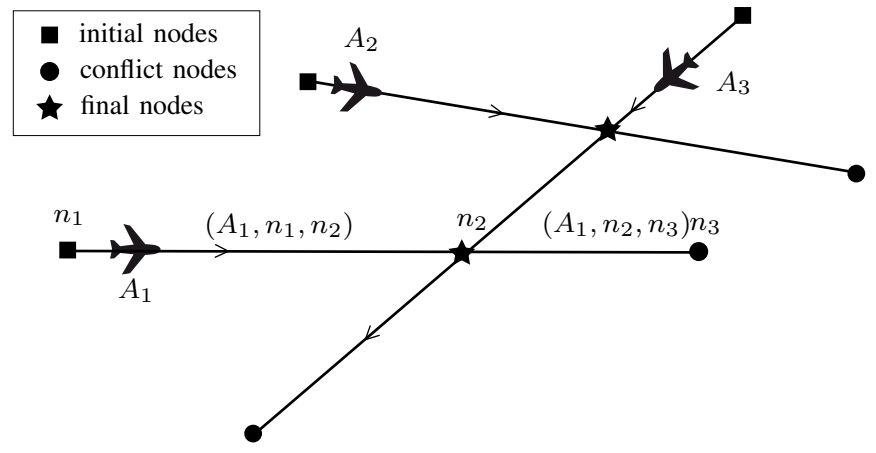

Fig. 2: Conflict graph for a situation with three aircraft [25]

the conflict point. Specifically, the times $A_{i}$ and $A_{j}$ fly over the conflict point must be separated with a minimum time separation $T_{i, j}^{\min }$ that depends on the velocities and on the angle between the trajectories of $A_{i}$ and $A_{j}$. If the velocities $V_{i}$ and $V_{j}$ are constant and so is the angle, $\theta_{i j}$, between the trajectories of $A_{i}$ and $A_{j}, T_{i, j}^{\min }$ may be computed as in [21]:

$$
T_{i, j}^{\min }=\frac{D}{V_{i} V_{j}\left|\sin \theta_{i, j}\right|} \sqrt{\left(V_{i}\right)^{2}+\left(V_{j}\right)^{2}-2 V_{i} V_{j} \cos \theta_{i, j}} .
$$

The combinatorics intrinsic to the problem appears in the choice of the aircraft that passes first at the conflict point. This is modeled with two big- $M$ constraints that involve one binary variable $\delta_{i j}$ and a large constant value $M$. The strength of this model is that it involves only one binary variable per pair of aircraft. Let $T_{i}$ and $T_{j}$ be the times $A_{i}$ and $A_{j}$ fly over the conflict point; then the separation constraints are

$T_{i}-T_{j} \geq T_{i, j}^{\min }-M \delta_{i j}$, and $T_{j}-T_{i} \geq T_{i, j}^{\min }-M\left(1-\delta_{i j}\right)$.

\section{B. Including heading maneuvers}

The maneuvers are restricted to those corresponding to a combination of the two patterns represented in Figure 3, and they cannot extend over more than one edge. In the heading maneuver depicted in Figure 3a, the aircraft has the same heading at the beginning and at the end of the maneuver: the purpose is to move the aircraft away laterally from its BT or to spatially recover it. The speed maneuvers illustrated in Figure $3 b$ end with the same speed as they started: their purpose is to move the aircraft back or forward temporally (or longitudinally) from its BT. These maneuvers are called trapezoidal speed and heading changes in reference to the shape of the graphs of speed and heading as functions of time. Finally, they are executed with the maximum yaw rate or acceleration to minimize the transition between the initial and final speeds or headings. The choice of these patterns is made to fit in the framework of MILPs. Other maneuvers may be more efficient, but the simplicity of the patterns may also be an asset for the communication between pilots and controllers.

The inclusion of heading maneuvers relies on the fact that a trapezoidal heading change generates a lateral shift with the BT. This shift can in turn be converted into a temporal shift at the conflict point. Indeed, Figure 4 illustrates that if $A_{i}$ is laterally shifted by $\Delta_{i}$ then the conflict point is moved by a distance $\Delta_{i} \tan \theta_{i j}$ along the trajectory of $A_{i}$,

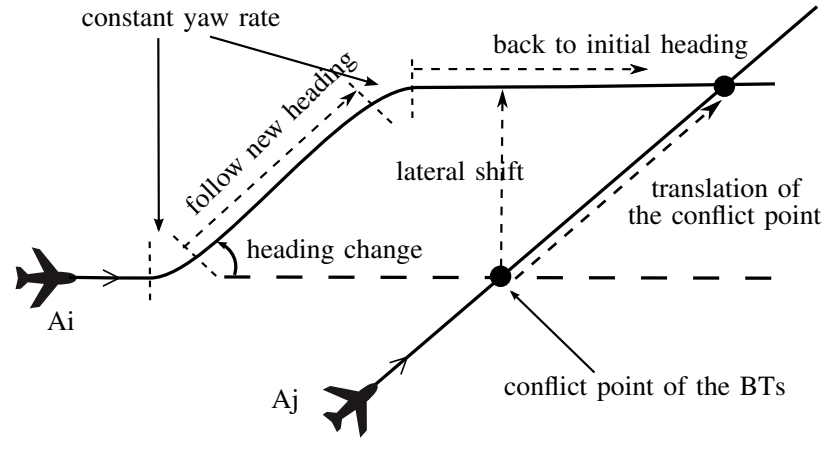

(a) Heading maneuver

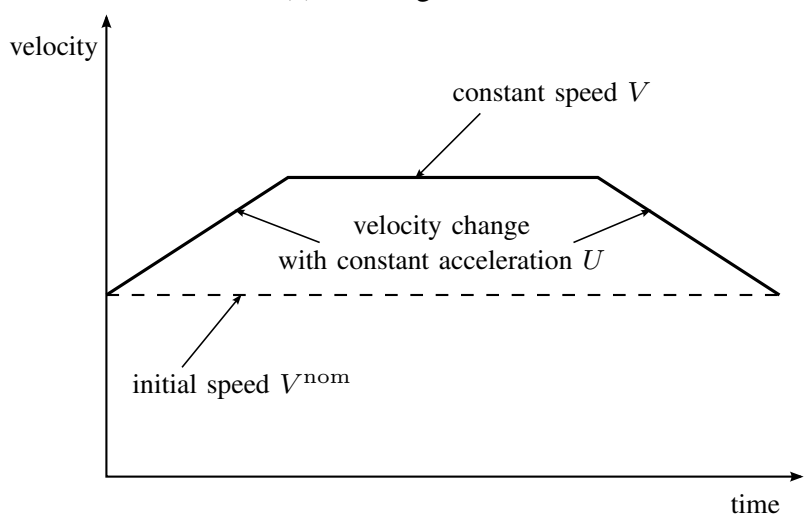

(b) Speed maneuver

Fig. 3: Representation of the two permitted types of maneuvers

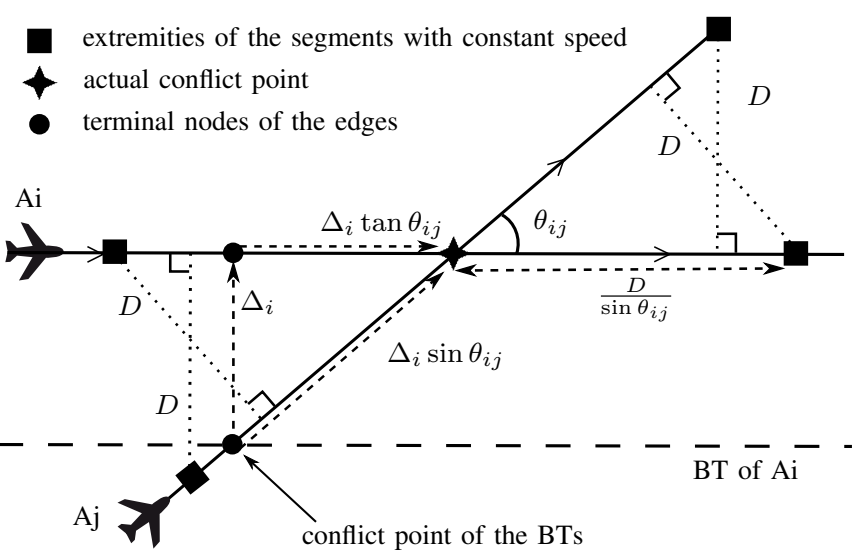

Fig. 4: Focus on a crossing conflict in presence of a lateral shift

and by a distance $\Delta_{i} \sin \theta_{i j}$ along the trajectory of $A_{j}$. If the speed vectors of $A_{i}$ and $A_{j}$ are constant, $T_{i}$ and $T_{j}$ are then linear functions of the decision variables. Constant speed vectors would forbid any maneuver, but it is actually not necessary to have the velocities constant in the region where there is no risk of conflict. As a consequence, the speed vectors are constrained to be constant on the segments where the distance between the trajectories is less than or equal to the required separation distance $D$. As depicted in Figure 4, this corresponds to an interval of length $2 \frac{D}{\sin \theta_{i j}}$ centered on the conflict point.

Finally, a stepwise linear approximation of the fuel con- 


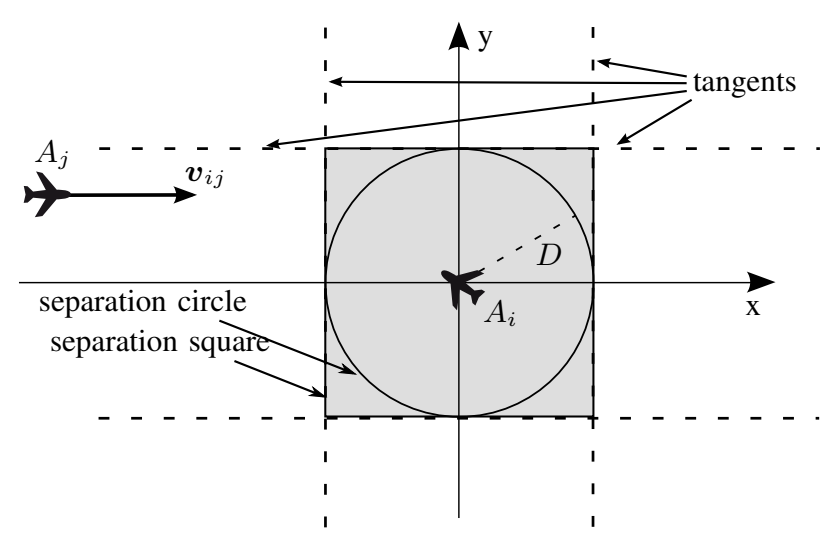

Fig. 5: Representation of the separation constraint

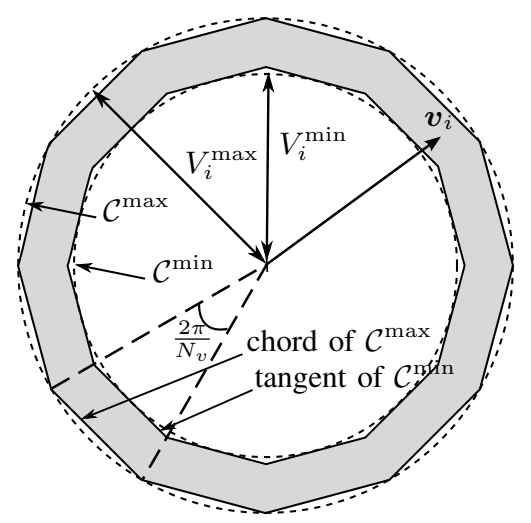

Fig. 6: Representation of the velocity bounds

sumption is minimized, and the recovery of the BT is ensured by demanding a zero lateral shift and minimizing the time shift at the nodes of $\mathcal{N}_{T}$. The overall model is called SPACE.

\section{TIME-DISCRETIZED MODEL}

Time-discretization consists in sampling the time horizon to model the problem with a set of variables and constraints that describe the states of the aircraft at each time step. This technique is used to derive an MILP in [13]-[16]. The model in [16] satisfies all the constraints described in Section II and differs only by the minimized objective, so we use it as a basis for the time-discretized model described in this section. As a consequence, we first present a synthesis of the study in [16]. We then develop an objective function that takes into account fuel consumption and delays.

\section{A. A linear time-discretized model}

In [16] the time interval $[0, T]$ is sampled according to a constant step $h$. A time-discretized model is then obtained by focusing on a sequence of $K+1$ times, $0=t_{0}<\ldots<$ $t_{K}=T$. Separation constraints and bounds on velocity and acceleration are nonlinear but they can be approximated using linear constraints.

The linearization relies on two related techniques that are most clearly understood via a geometric representation of the constraints. Figure 5 illustrates a potential conflict between two aircraft $A_{i}$ and $A_{j}$ in the mobile frame attached to $A_{i}$. The loss of separation is avoided if $A_{j}$ remains outside of the separation circle with radius $D$ centered on $A_{i}$. The circle is then approximated using four tangents (the dotted lines) that delimit a separation square. Each tangent corresponds to one linear constraint and at least one of these constraints must be satisfied. As in SPACE, this disjunction is modeled with a set of big-M constraints involving as many binary variables as tangents: $\forall\left(A_{i}, A_{j}\right) \in \mathcal{C}, \forall k \in\{0, \ldots, K-1\}$ :

$$
\begin{aligned}
& \left\langle\boldsymbol{p}_{i j}^{k} \mid \boldsymbol{e}_{\theta}\right\rangle \geq D+h^{2} / 8\left(\bar{u}_{i}^{k}+\bar{u}_{j}^{k}\right)-M \delta_{i j}^{k \theta}, \forall \theta \in \Theta_{s} \\
& \left\langle\boldsymbol{p}_{i j}^{k+1} \mid \boldsymbol{e}_{\theta}\right\rangle \geq D+h^{2} / 8\left(\bar{u}_{i}^{k}+\bar{u}_{j}^{k}\right)-M \delta_{i j}^{k \theta}, \forall \theta \in \Theta_{s} \\
& \sum_{\theta \in \Theta_{s}} \delta_{i j}^{k \theta}=3
\end{aligned}
$$

where $\boldsymbol{p}_{i j}^{k}$ is the relative position of $A_{j}$ with respect to $A_{i}$ at $t_{k}, \Theta_{s}=\left\{0, \frac{\pi}{2}, \pi, \frac{3 \pi}{2}\right\}$ identifies the positions of the tangents, $\delta_{i j}^{k \theta}$ is a binary variable that nullifies only if the corresponding contraints (6)-(7) are satisfied, $\bar{u}_{i}^{k}$ is an upper bound of the acceleration of $A_{i}$ at $t_{k}, \boldsymbol{e}_{\theta}$ is the unit vector with coordinates $(\cos \theta, \sin \theta)$ and $\langle. \mid$.$\rangle is the scalar product. Constraint (8)$ guarantees that at least one of the four tangent constraints is satisfied. The two constraints (6)-(7) are necessary to guarantee that the separation is maintained on the whole $\left[t_{k}, t_{k+1}\right]$ time interval. The higher order terms involving $\bar{u}_{i}^{k}$ and $\bar{u}_{j}^{k}$ are necessary to take the acceleration into account in noninstantaneous maneuvers.

Figure 6 represents the bounds on velocity as two concentric circles in $\mathcal{C}^{\min }$ and $\mathcal{C}^{\max }$ with radii $V_{i}^{\min }$ and $V_{i}^{\max }$ (in dotted lines). To respect the bounds, the endpoint of $\boldsymbol{v}_{i}$ must lie between $\mathcal{C}^{\text {min }}$ and $\mathcal{C}^{\text {max }}$. The circles $\mathcal{C}^{\text {min }}$ and $\mathcal{C}^{\text {max }}$ are then approached using respectively $N_{v}$ tangents and chords. With this approximation, $\boldsymbol{v}_{i}$ must lie in the shaded area of Figure 6. This surface is included between $\mathcal{C}^{\min }$ and $\mathcal{C}^{\text {max }}$, so the original bounds will also be respected. The upper bound is then a conjunction of linear constraints and the lower bound is a disjunction modeled with big-M constraints: $\forall A_{i} \in \mathcal{A}, \forall k \in\{0, \ldots, K\}$ :

$$
\begin{aligned}
\left\langle\boldsymbol{v}_{i}^{k} \mid \boldsymbol{e}_{\theta}\right\rangle & \leq V_{i}^{\max } \times \cos \left(\pi / N_{v}\right), \forall \theta \in \Theta_{v} \\
\left\langle\boldsymbol{v}_{i}^{k} \mid \boldsymbol{e}_{\theta}\right\rangle & \geq V_{i}^{\min }-M_{v} \epsilon_{i}^{k \theta}, \forall \theta \in \Theta_{v} \\
\sum_{\theta \in \Theta_{v}} \epsilon_{i}^{k \theta} & =N_{v}-1,
\end{aligned}
$$

where $\boldsymbol{v}_{i}^{k}$ is the speed vector of $A_{i}$ at $t_{k}, \Theta_{v}=\left\{\frac{2 n \pi}{N_{v}}\right\}_{0 \leq n<N_{v}}$, $\epsilon_{i}^{k \theta}$ is a binary variable that nullifies only if the corresponding constraint is satisfied and $M_{v}$ is a large constant value.

\section{B. Minimizing a meaningful objective function}

The model in [16] minimizes acceleration and requires a complete recovery of the BTs. Our intent is to relax the time recovery and minimize a combination of fuel consumption and delay.

The fuel consumption per time unit is a nonlinear function of velocity that can be linearized if we make the following two approximations. First, the velocity is approximated on each subinterval $\left[t_{k}, t_{k+1}\right]$ by the average value 


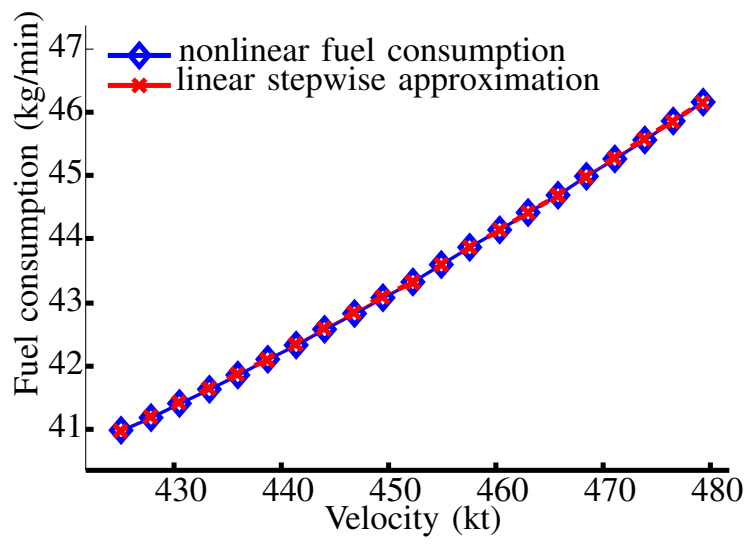

Fig. 7: Fuel consumption per time unit: original function and stepwise approximation for an Airbus A320 at 33,000 feet

$\frac{1}{2}\left(\left\|\boldsymbol{v}_{i}^{k}\right\|+\left\|\boldsymbol{v}_{i}^{k+1}\right\|\right)$. Second, the fuel consumption is approximated with a stepwise linear function joining $N_{Z}+1$ points of the real curve. The accuracy of the second approximation is illustrated in Figure 7 where the fuel consumption of an Airbus A320 is approached with as few as four segments.

For a particular aircraft $A_{i} \in \mathcal{A}$, let $Z=\alpha_{i}^{n} V+\beta_{i}^{n}$ be the equation of the $n^{\text {th }}$ segment, $1 \leq n \leq N_{Z}$. The fuelconsumption function was found to be convex on the interval $\left[V^{\min }, V^{\max }\right]$ for all the aircraft types that were tested, so it is approximated by $z_{i}^{k}$ on $\left[t_{k}, t_{k+1}\right]$ if $z_{i}^{k}$ is minimized and

$$
z_{i}^{k} \geq h \times\left(\frac{\alpha_{n}}{2}\left(\left\|\boldsymbol{v}_{i}^{k}\right\|+\left\|\boldsymbol{v}_{i}^{k+1}\right\|\right)+\beta_{n}\right), 1 \leq n \leq N_{Z}
$$

Now, $\left\|\boldsymbol{v}_{i}^{k}\right\|$ is a nonlinear function of the speed coordinates but it may be approximated with a new variable $\bar{v}_{i}^{k}$ by replacing the upper bound (9) with:

$$
\begin{aligned}
& \left\langle\boldsymbol{v}_{i}^{k} \mid \boldsymbol{e}_{\theta}\right\rangle \leq \bar{v}_{i}^{k} \cos \left(\pi / N_{v}\right), \forall \theta \in \Theta_{v} \\
& V_{i}^{\min } \leq \bar{v}_{i}^{k} \leq V_{i}^{\max }
\end{aligned}
$$

These constraints guarantee that the velocity remains below $V_{i}^{\max }$ and $\bar{v}_{i}^{k} \geq\left\|\boldsymbol{v}_{i}^{k}\right\|$. As a consequence, instead of (12) we add the following linear constraints to the model:

$$
z_{i}^{k} \geq h \times\left(\frac{\alpha_{n}}{2}\left(\bar{v}_{i}^{k}+\bar{v}_{i}^{k+1}\right)+\beta_{n}\right), 1 \leq n \leq N_{Z} .
$$

Since $z_{i}^{k}$ is minimized, the upper bound $\bar{v}_{i}^{k}$ will get as close as possible to the actual velocity $\left\|\boldsymbol{v}_{i}^{k}\right\|$.

Space recovery is then enforced by constraint (5), and the delay is minimized by penalizing the longitudinal deviations:

$$
\begin{aligned}
& \sum_{A_{i} \in \mathcal{A}} \sum_{t_{k} \in \mathcal{T}^{-}} z_{i}^{k}+ \\
& \quad\left(\Delta_{\|, i}^{+}+\Delta_{\|, i}^{-}\right) \times C_{d, i}\left(V_{i}^{\mathrm{nom}}\right)+\rho_{i}^{+} \Delta_{\|, i}^{+}+\rho_{i}^{-} \Delta_{\|, i}^{-},
\end{aligned}
$$

where $\Delta_{\|, i}^{+}$and $\Delta_{\|, i}^{+}$are the positive and negative longitudinal deviations of $A_{i}$.

The overall time-discretized model is called TIME.

\section{THE ONE-MANEUVER SIMPLIFICATION}

The specificity of the model presented in this section is that it describes a conflict resolution involving only one maneuver

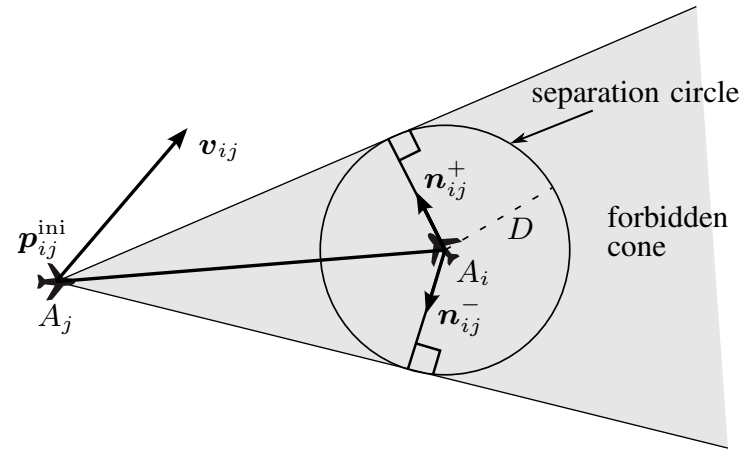

Fig. 8: Conflict solved with only one instantaneous maneuver

for each aircraft, all these maneuvers being executed simultaneously at the initial time. This model, denoted ONE, is based on that developed by Vela et al. [18] in which fuel consumption is also minimized. To respect the specific constraints listed in Section II, we made three major modifications to the model of [18]. First, the maneuvers are not instantaneous. This allows to consider the bounds of acceleration and yaw rate and reduce the error made in the computation of the maneuvers. Second, a recovery maneuver is introduced to compute the total fuel consumption and treat the recovery of the BT. Finally, the model is solved multiple times with a receding horizon to allow for the same number of maneuvers as in TIME.

We describe the constraints and the objective function of ONE in Sections V-A and V-B, respectively. The receding horizon algorithm implemented to compute multiple maneuvers is then detailed in Section $\mathrm{V}-\mathrm{C}$.

\section{A. Constraints of the simplified model}

The maneuvers are assumed to be performed with a constant acceleration vector during a given time step. For a fair comparison, the length of the time step is set to the sampling period $h$ of TIME. Starting with known position, speed vector and velocity, $\boldsymbol{p}_{i}^{\text {ini }}, \boldsymbol{v}_{i}^{\text {ini }}$ and $V_{i}^{\text {ini }}$, the movement of an aircraft $A_{i}$ is thus entirely described by the target speed vector $\boldsymbol{v}_{i}$ that is reached at time $h$.

Adapting notations of the previous section to a unique time step, the constraints on velocity are then similar to (10)-(11) and (13)-(14): $\forall A_{i} \in \mathcal{A}$ :

$$
\begin{aligned}
& \left\langle\boldsymbol{v}_{i} \mid \boldsymbol{e}_{\theta}\right\rangle \leq \bar{v}_{i} \times \cos \left(\pi / N_{v}\right), \forall \theta \in \Theta_{v} \\
& V_{i}^{\min } \leq \bar{v}_{i} \leq \min \left(V_{i}^{\max }, V_{i}^{\mathrm{ini}}+h U_{i}^{\max }\right) \\
& \left\langle\boldsymbol{v}_{i} \mid \boldsymbol{e}_{\theta}\right\rangle \geq \max \left(V_{i}^{\min }, V_{i}^{\mathrm{ini}}-h U_{i}^{\max }\right)-M_{v} \epsilon_{i}^{\theta}, \theta \in \Theta_{v} \\
& \sum_{\theta \in \Theta_{v}} \epsilon_{i}^{\theta}=N_{v}-1
\end{aligned}
$$

One effect of the simplification is that upper bounds on the acceleration could be included in the constraints on velocity (18) and (19), because the initial speed is known. The upper bound on the yaw rate can also be expressed with linear constraints involving the upper bound on the velocity, $\bar{v}_{i}$ :

$$
\left\langle\boldsymbol{v}_{i} \mid \boldsymbol{v}_{i}^{\mathrm{ini}}\right\rangle \geq \bar{v}_{i} \times V_{i}^{\mathrm{ini}} \times \cos \left(h \omega_{i}^{\max }\right), \forall A_{i} \in \mathcal{A} .
$$

The most significant effect of performing only one maneuver is that the separation constraint becomes a disjunc- 
tion between two linear constraints if the speed changes are instantaneous. Figure 8 shows a potential conflict between two aircraft $A_{i}$ and $A_{j}$ in the mobile frame attached to $A_{i}$. Vector $\boldsymbol{v}_{i j}$ is the target relative speed vector resulting from the maneuvers of $A_{i}$ in $A_{j}$. Geometrically, the conflict is solved if $A_{j}$ remains outside the separation circle. Since only one maneuver is performed, an equivalent condition is that $\boldsymbol{v}_{i j}$ is outside the cone delimited by the two tangents going through the initial relative position. The remaining issue is that speed changes are not instantaneous.

Proposition 1. Consider $\boldsymbol{p}_{0}, \boldsymbol{v}_{0}, \boldsymbol{u}_{0} \in \mathbb{R}^{2}$ and the functions $\boldsymbol{p}$ and $\widetilde{\boldsymbol{p}}$ defined by

$$
\begin{aligned}
& \boldsymbol{p}(t)=\left\{\begin{array}{l}
\boldsymbol{p}_{0}+t \boldsymbol{v}_{0}+\frac{t^{2}}{2} \boldsymbol{u}_{0}, 0 \leq t \leq h \\
\boldsymbol{p}_{0}+h \boldsymbol{v}_{0}+\frac{h^{2}}{2} \boldsymbol{u}_{0}+(t-h)\left(\boldsymbol{v}_{0}+\boldsymbol{u}_{0} h\right), h \leq t
\end{array}\right. \\
& \widetilde{\boldsymbol{p}}(t)=\left\{\begin{array}{l}
\boldsymbol{p}_{0}+t \boldsymbol{v}_{0}, 0 \leq t \leq \frac{h}{2} \\
\boldsymbol{p}_{0}+\frac{h}{2} \boldsymbol{v}_{0}+\left(t-\frac{h}{2}\right)\left(\boldsymbol{v}_{0}+\boldsymbol{u}_{0} h\right), \frac{h}{2} \leq t .
\end{array}\right.
\end{aligned}
$$

Then, $\boldsymbol{p}(t)=\widetilde{\boldsymbol{p}}(t), \forall t \geq h$.

Proposition 1 states that after a speed vector change with constant acceleration, the position is the same as if the change had been achieved instantaneously after a delay equal to $h / 2$. Moreover, $h$ is typically set to less than one minute, so a loss of separation predicted in the time interval $[0, h]$ would be treated with a specific emergency protocol. As a consequence, it is sufficient to consider the conflicts that are detected after time $h$. Everything then behaves as if maneuvers were instantaneous and the initial positions of the aircraft were given by $\widetilde{\boldsymbol{p}}_{i}^{\mathrm{ini}}=$ $\boldsymbol{p}_{i}^{\text {ini }}+\frac{h}{2} \boldsymbol{v}_{i}^{\text {ini }}$. For a given pair of aircraft $\left(A_{i}, A_{j}\right)$, the tangents defining the forbidden cone in Figure 8 are computed using $\widetilde{\boldsymbol{p}}_{i}^{\text {ini }}$ and $\widetilde{\boldsymbol{p}}_{j}^{\text {ini }}$ as the initial positions of the two aircraft. The two unit vectors, $\widetilde{\boldsymbol{n}}_{i j}^{+}$and $\widetilde{\boldsymbol{n}}_{i j}^{-}$, orthogonal to these tangents and pointing outside the forbidden cone then give rise to the separation constraints:

$$
\begin{aligned}
& \left\langle\boldsymbol{v}_{j}-\boldsymbol{v}_{i} \mid \tilde{\boldsymbol{n}}_{i, j}^{+}\right\rangle \geq-M \delta_{i j}, \forall\left(A_{i}, A_{j}\right) \in \mathcal{C}, \\
& \left\langle\boldsymbol{v}_{j}-\boldsymbol{v}_{i} \mid \widetilde{\boldsymbol{n}}_{i, j}^{-}\right\rangle \geq-M\left(1-\delta_{i j}\right), \forall\left(A_{i}, A_{j}\right) \in \mathcal{C},
\end{aligned}
$$

with $\delta_{i j} \in\{0,1\}$. The value of $M$ must be such that, for all valid values of $\boldsymbol{v}_{i}$ and $\boldsymbol{v}_{j}$, (22) and (23) are respectively satisfied if $\delta_{i j}=1$ and $\delta_{i j}=0$. If we solve the model with a branch and bound method based on linear relaxations of the MILP, the best value of $M$ is the smallest value that satisfies this condition, because it leads to the tightest linear relaxations. Here, we notice that

$$
\left\langle\boldsymbol{v}_{j}-\boldsymbol{v}_{i} \mid \widetilde{\boldsymbol{n}}_{i, j}^{+}\right\rangle \geq-\left(\left\|\boldsymbol{v}_{i}\right\|+\left\|\boldsymbol{v}_{j}\right\|\right)
$$

A valid value of the constant is thus obtained by $M=V_{i}^{\max }+$ $V_{j}^{\max }$. It is also the best value if we do not take maximum accelerations and yaw rates into account.

Another application of Proposition 1 is to compute the maximum error that would be made with the assumption of instantaneous maneuvers. An equivalent formulation of the proposition is that instantaneous maneuvers neglect an $h / 2$ delay in the starting time of the maneuver. The largest maneuver that can be performed corresponds to an acceleration $h U^{\max }$ and a turn $h \omega^{\max }$. Denoting $\boldsymbol{v}_{i}^{\max }$ the resulting target speed vector, the $h / 2$ delay can then create at most a shift $h / 2\left(\boldsymbol{v}_{i}^{\text {ini }}-\boldsymbol{v}_{i}^{\max }\right)$ in the position of $A_{i}$. With standard numerical values (see Table I), the maximum error is equal to $0.85 \mathrm{NM}$. Considering that the same error can be done for $A_{j}$ and that the standard required distance is $5 \mathrm{NM}$, this confirms that the maneuvers should not be considered instantaneous.

\section{B. Minimizing fuel consumption and delay}

The estimation of the fuel consumption and delay rely on the description of a complete trajectory. It is thus necessary that the aircraft revert to their BTs. The difficulty is that an aircraft should not start a recovery maneuver while a loss of separation is possible. The value of the instant when a conflict ends is a nonlinear function of the speed and acceleration vectors of the aircraft. However, if no maneuver is performed, it is possible to find an upper bound of this instant that depends only on the initial data.

Proposition 2. Let $\left(A_{i}, A_{j}\right) \in \mathcal{C}$ be such that the two aircraft fly with constant speed vectors $\boldsymbol{v}_{i}^{\text {ini }}$ and $\boldsymbol{v}_{j}^{\text {ini }}$. Assume that $A_{i}$ and $A_{j}$ are separated at $t=0$ and $t=T$, and that a loss of separation occurs in $[0, T]$. The loss of separation ends no later than at $t=\tau_{i j}$ with

$$
\tau_{i j}=\frac{D}{\left\|\boldsymbol{v}_{i j}^{\mathrm{ini}}\right\|}-\left\langle\boldsymbol{p}_{i j}(0) \mid \frac{\boldsymbol{v}_{i j}^{\mathrm{ini}}}{\left\|\boldsymbol{v}_{i j}^{\mathrm{ini}}\right\|^{2}}\right\rangle .
$$

Proof. For constant speed vectors $\boldsymbol{p}_{i j}(t)=\boldsymbol{p}_{i j}(0)+t \boldsymbol{v}_{i j}^{\text {ini }}$, $t \in[0, T]$. The distance between the two aircraft at time $t$ is bounded by

$\left\|\boldsymbol{p}_{i j}(t)\right\| \geq\left\langle\boldsymbol{p}_{i j}(t) \mid \frac{\boldsymbol{v}_{i j}^{\mathrm{ini}}}{\left\|\boldsymbol{v}_{i j}^{\text {ini }}\right\|}\right\rangle=\left\langle\boldsymbol{p}_{i j}(0) \mid \frac{\boldsymbol{v}_{i j}^{\text {ini }}}{\left\|\boldsymbol{v}_{i j}^{\text {ini }}\right\|}\right\rangle+t\left\|\boldsymbol{v}_{i j}^{\text {ini }}\right\|$

Replacing $t$ with the expression of $\tau_{i j}$ proves the result.

Proposition 2 shows that if no maneuver is performed, an aircraft $A_{i}$ will not be involved in a loss of separation after $\tau_{i}=\max \left\{\tau_{i j}:\left(A_{i}, A_{j}\right) \in \mathcal{C}\right\}$. Clearly, the estimation of the end of the conflicts is incorrect if maneuvers are performed. Nevertheless, it remains a valid guess as long as it is not used to compute a real maneuver. A second virtual maneuver starting at $\tau_{i}$ is thus added to simulate the complete trajectory for $A_{i}$. This speed change is included only to estimate the overall cost of a maneuver and is not meant to lead to an actual control instruction. The actual recovery maneuver should be started only once it cannot create a loss of separation.

Let $\boldsymbol{v}_{i}^{\prime}, A_{i} \in \mathcal{A}$, be the target speed vectors of the virtual recovery maneuvers. According to Proposition 1, the estimated final position is equivalently reached by following $\boldsymbol{v}_{i}$ during $\tau_{i}$ and $\boldsymbol{v}_{i}^{\prime}$ until the end of the time horizon:

$$
\boldsymbol{p}_{i}(T)=\widetilde{\boldsymbol{p}}_{i}^{\mathrm{ini}}+\tau_{i} \boldsymbol{v}_{i}+\left(T-\tau_{i}-\frac{h}{2}\right) \boldsymbol{v}_{i}^{\prime}, \forall A_{i} \in \mathcal{A} .
$$

As for TIME, the fuel consumption is approximated with a stepwise linear function, and the final position of each aircraft $A_{i}$ is used to compute the longitudinal gaps $\Delta_{\|, i}^{+}$and $\Delta_{\|, i}^{-}$. The associated constraints and objective function are then similar to (15) and (16); they are omitted to save space. 


\section{Building a complete trajectory}

Solving ONE produces at most one actual speed change for each aircraft. For a consistent comparison with TIME, ONE is solved at each time step $t_{k} \in \mathcal{T}$ according to the receding-horizon procedure summarized in Algorithm 1. The result is a set of complete conflict-free trajectories reverting to the associated BTs. In Algorithm 1, the conditional loop on lines 4-6 identifies the moment when the recovery maneuver may be started. If $\tau_{i} \leq t_{k}$, then no maneuver is needed to solve the conflicts involving $A_{i}$, so it may revert to its $\mathrm{BT}$. The recovery maneuver is computed to minimize a combination of the fuel consumption and the penalties due to the longitudinal deviation, as in (16).

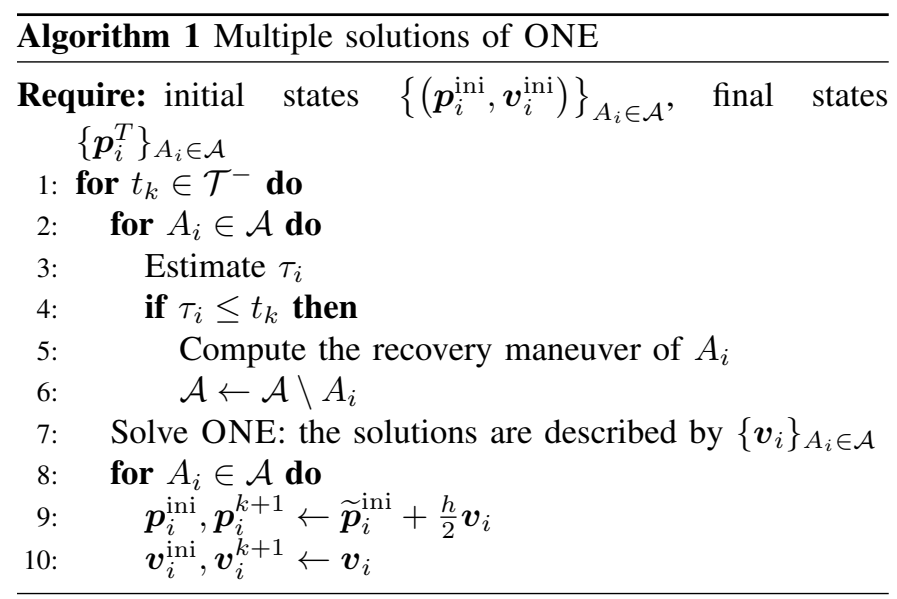

One drawback of Algorithm 1 is that the first solution of ONE computes a set of simultaneous maneuvers that resolve every conflict. Stated otherwise, there is no possibility to postpone the resolution of a conflict if needed. In contrast, the maneuvers may be started at any time step in TIME an on any edge in SPACE. However, in a deterministic environment, conflicts may be solved with smaller maneuvers if the latter are started as soon as possible. Simultaneous maneuvers should be efficient if they are started at $t=0$.

In the remainder of this article, the term ONE will refer to the model described in the previous subsection or to Algorithm 1, provided the context is unambiguous.

\section{COMPUTATIONAL EXPERIMENTS}

In this section, we compare the three MILPs described in the previous sections. In Section VI-A, we compare the dimensions of the three formulations from a theoretical point of view. In Section VI-B, we then describe the benchmark used in the experimental tests, and we analyze the results in Section VI-C.

\section{A. Comparison of the three formulations}

The models TIME, ONE, and SPACE share an essential feature: their feasible solutions all satisfy the constraints of the problem definition in Section II. Hence, the approximations that were made to obtain MILPs all lead to more conservative constraints than the original ones. As a consequence, the differences between the three models impact only the computational time and the cost of the optimal solution.
The three models involve a combinatorial decision. For each pair of aircraft, a disjunction of linear constraints guarantees the separation, so the solution process needs to choose which constraint to satisfy. In SPACE, two choices are available for each pair of aircraft, so $2^{|\mathcal{C}|}$ configurations are possible. The same is true for ONE, but the model is solved at each time step, so the solution process may explore $K \times 2^{|\mathcal{C}|}$ configurations. In TIME, the separation must be ensured at each time step by respecting one of the $N_{\delta}$ tangent constraints, which leads to $\left(N_{\delta}\right)^{K \times|\mathcal{C}|}$ possibilities. The largest roundabout instance described in the next section gives rise to $7 \times 10^{9}$ configurations for SPACE, $7 \times 10^{10}$ for ONE and $5 \times 10^{33}$ for TIME. No fast solution process would explore all the configurations, but this provides a hierarchy that would abstract to any set of models built with the same discretizations.

The remaining variables and constraints of TIME describe the state of each aircraft at each time step. Since ONE is a time decomposition of TIME, the conflict resolution with ONE involves $K$ solutions of a model with $O(|\mathcal{A}|)$ variables and constraints whereas TIME includes $O(K \times|\mathcal{A}|)$ variables and constraints. The comparison with SPACE is more fuzzy. Indeed, SPACE computes the states of the aircraft on each edge of the conflict graph, and the relation between the numbers of aircraft and edges depends a lot on the considered instance. Moreover, the approximations involved in the linearizations of TIME and SPACE are different. The corresponding parameters may thus impact both the solution times and the optimal costs. For instance, if a larger number of tangents are used to approach the separation circle in TIME, the solution cost may be improved, but the solution time will be longer.

\section{B. Generation of a large benchmark of complex problems}

The experimental comparison of TIME, ONE, and SPACE is based on the benchmark of [25], slightly extended to include larger instances. The instances implement the three global schemes depicted in Figure 9. For each pattern, we consider five to six scenarios that mostly differ in the number of aircraft involved. The scenarios are thus denoted by the first letter of the corresponding pattern and the number of aircraft. The roundabout scenarios (R04-R08) involve 4 to 8 aircraft initially positioned in a circle and heading towards its center. In the trail crossing scenarios (T02-T12), two trails of 2 to 6 aircraft intersect with a varying angle. The grid scenarios (G06-G12) oppose two pairs of trails of 1 to 3 aircraft that intersect orthogonally. These patterns represent complex situations in which a large number of losses of separation are predicted to occur in the next five to ten minutes. As the number of aircraft grows, the corresponding situation involves much more potential conflicts than the most difficult situations that a controller ever deals with. Although they cannot reflect the complexity of real traffic, these academic instances allow to test the three algorithms with a diverse set of potential conflicts and explore their limits.

Each scenario is used to randomly generate 100 instances. When we generate the instances randomly, the initial separation between aircraft flying on the same trail is set to 
$8+U([-2.5,2.5]) \mathrm{NM}$, where $U([a, b])$ follows a uniform random law on the interval $[a, b]$. The distance between the first aircraft of each trail and the closest conflict point is also perturbed with a uniform term $U([-2.5,2.5]) \mathrm{NM}$, and the crossing angle $\alpha$ follows $U\left(\left[\frac{\pi}{4}, \frac{3 \pi}{4}\right]\right)$ for the trail scenarios.

The values of the parameters of the models are listed in Table I. They reflect the content of BADA [31] for an Airbus A320 flying at 33,000 feet. They also ensure the comfort of passengers, according to the study of Paielli [29]. Additionally, the characteristic values of the linear approximations are set to $N_{Z}=4$ and $N_{v}=36$.

\begin{tabular}{cccccccc}
\hline$D$ & $h$ & $K$ & $V^{\text {nom }}$ & $V^{\min }$ & $V^{\max }$ & $U^{\max }$ & $\omega^{\max }$ \\
\hline $5 \mathrm{NM}$ & $30 \mathrm{~s}$ & 20 & $452 \mathrm{kt}$ & $425 \mathrm{kt}$ & $479 \mathrm{kt}$ & $0.4 \mathrm{kt} . \mathrm{s}^{-1}$ & $0.88^{\circ} . \mathrm{s}^{-1}$ \\
\hline
\end{tabular}

TABLE I: Optimization and aircraft-performance parameters

\section{Analysis of the results}

We solve TIME, ONE, and SPACE on 1600 instances generated randomly according to sixteen scenarios. Gurobi is used with its default options to solve each MILP on a quadcore $2.5-\mathrm{GHz}$ Intel processor with $4 \mathrm{~Gb}$ of RAM. The time limit of Gurobi is set to $120 \mathrm{~s}$, and we keep the best available solution when the limit is reached. The simulation results are summarized in Figure 10. For each scenario, Figure 10a gives the fractions of instances for which conflict-free solutions are found, and Figures $10 \mathrm{~b}$ and 10c give the average costs per aircraft and the Gurobi runtimes. The average costs are given in kilograms of fuel because the delay penalties convert the time deviations into fuel consumptions. Moreover, they take into account only the conflict-free solutions, because the costs of infeasible solutions may be exceptionally high. We also emphasize that the runtime given for ONE reflects the $K$ solutions of the model, as described in Algorithm 1. The scenarios are ordered on the $\mathrm{x}$-axis according to the growing solution time of SPACE, which is a valid measure of complexity.

Although the three graphs in Figure 10 reveal significant differences between the three models, we draw the attention of the reader to the overall efficiency of the three algorithms. If we omit G12, R07, and R08, which are included primarily to test the limits of the models, a conflict-free solution is found for all the instances. The computational time is always reasonable and is not far from being compatible with an operational implementation. Moreover, the average cost of the associated maneuvers does not exceed $10 \mathrm{~kg}$ per aircraft.

When we compare the curves, a gross ordering appears, with only minor exceptions if we consider G12, R07, or R08. Let $|\mathcal{S}|_{\text {MOD }}, Z_{\mathrm{MOD}}$, and $\mathrm{cpu}_{\mathrm{MOD}}$ be respectively the fraction of conflict-free solutions, the average cost, and the average computational time when solving MOD. Global relations exist between the three models:

$$
\begin{array}{r}
|\mathcal{S}|_{\mathrm{SPACE}} \leq|\mathcal{S}|_{\mathrm{TIME}} \leq|\mathcal{S}|_{\mathrm{ONE}} \\
\mathrm{Z}_{\mathrm{SPACE}} \geq \mathrm{Z}_{\mathrm{TIME}} \geq \mathrm{Z}_{\mathrm{ONE}} \\
\mathrm{cpu}_{\mathrm{TIME}} \geq \mathrm{cpu}_{\mathrm{ONE}} \geq \mathrm{cpu}_{\mathrm{SPACE}} .
\end{array}
$$

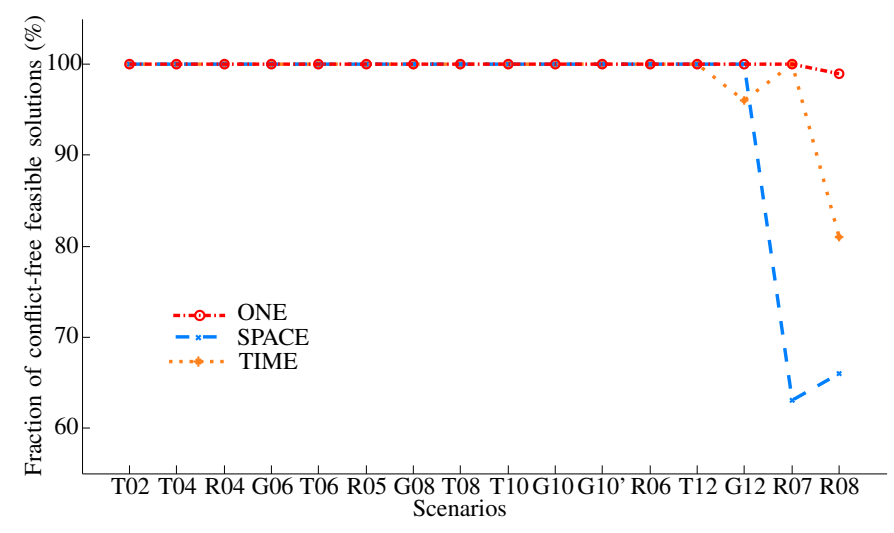

(a) Number of feasible solutions

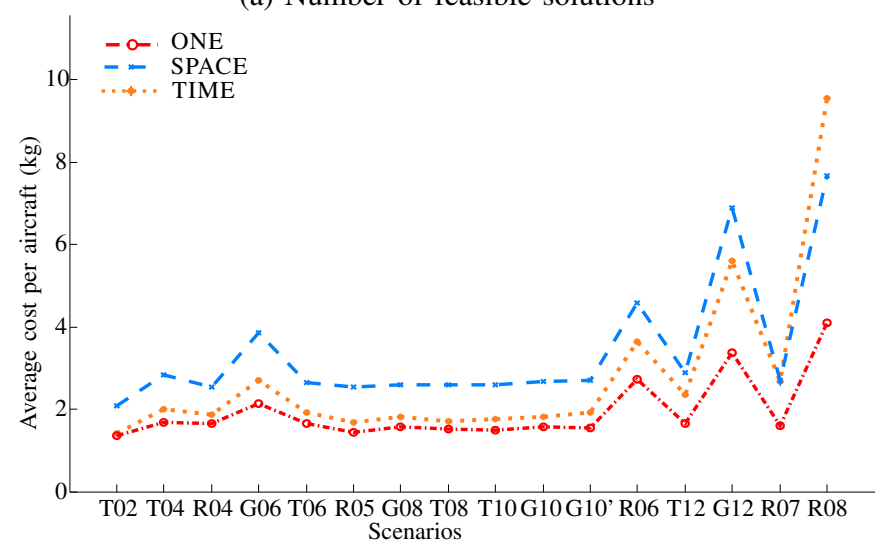

(b) Average cost per aircraft of feasible solutions

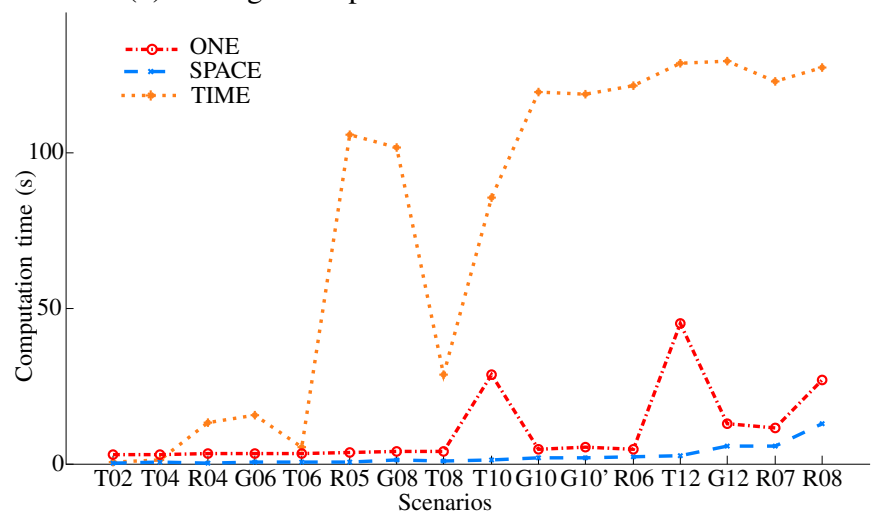

(c) Average computation time

Fig. 10: Comparison of the three mixed integer linear models

The discussion about complexity in Section VI-A allows to explain (26). The model ONE is a decomposition of TIME, so it needs to be solved multiple times, but each call to Gurobi takes a negligible portion of the runtime needed for TIME. We also observe that the solution time of SPACE is in the same order of magnitude as that of ONE, so the order might be reversed for slightly different modeling choices.

To explain (25), we consider a simple instance involving two aircraft whose planned trajectories collide (i.e. reach a $0 \mathrm{NM}$ separation) at $t=4 \mathrm{~min}$. The angle between the trajectories is equal to $90^{\circ}$, and the required separation distance is increased to $10 \mathrm{NM}$ to distinguish the solutions more easily. Figure 11 displays the trajectories found by the three models in the 


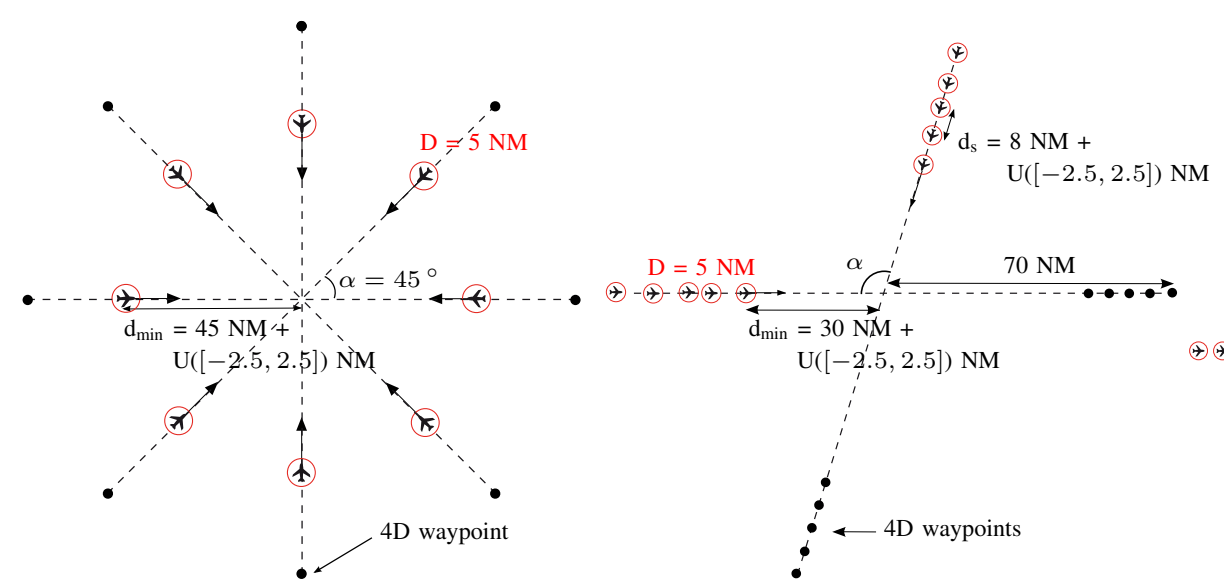

(a) Roundabout scenario (b) Trail crossing scenario

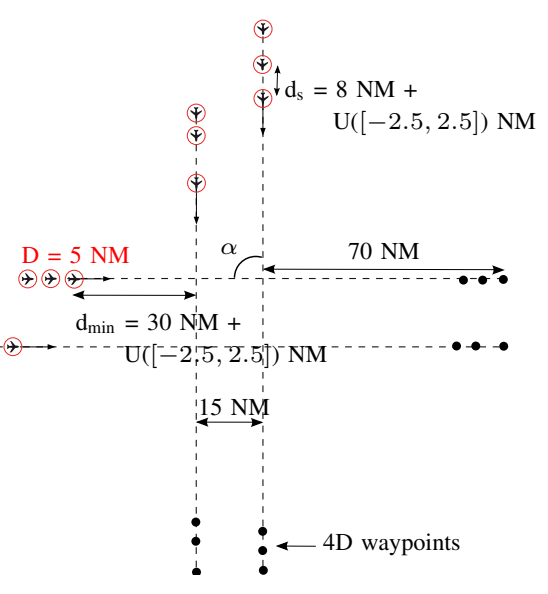

(c) Grid scenario

Fig. 9: Scenarios used to build the benchmark

mobile frame attached to one of the aircraft. Since the three trajectories were hard to read on a single figure, they appear on the two separate figures. The costs of the solutions follow the order in $(25): \mathrm{Z}_{\mathrm{SPACE}} \approx 8 \mathrm{~kg}, \mathrm{Z}_{\mathrm{TIME}} \approx 10.5 \mathrm{~kg}$ and $\mathrm{Z}_{\mathrm{ONE}} \approx 12 \mathrm{~kg}$. Figure $11 \mathrm{a}$ shows why TIME generates a more expensive solution than ONE. In TIME, the separation circle is approached with a square, so the relative trajectory of $A_{j}$ goes farther from $A_{i}$ than needed. In contrast, the trajectory generated by ONE is tangent to the circle. Figure $11 \mathrm{~b}$ shows that the main drawback of SPACE is that it requires the speed vectors to be constant in the vicinity of the conflict point. Therefore, the lateral shift is created with a larger heading change than needed.

To explain the ordering in (24), we first focus on the curve of SPACE in Figure 10a. Speed vectors need to be constant on intervals centered on the conflict points in SPACE. This reduces the space available for the maneuvers. Since all the potential conflicts occur around the same point in the roundabout scenarios, there is finally insufficient space to avoid every loss of separation in R07 and R08. To a lesser extent, restricting the maneuvers of SPACE to two fixed patterns also reduces the space of the conflict-free solutions. As a consequence, if the collision was planned at $t=3$ minutes instead of 4 minutes in the example depicted in Figure 11b, SPACE would be the only model unable to produce conflict-free trajectories. Secondly, the curve of TIME in Figure 10a shows that no conflict-free trajectories could be found for some instances of G-12 and R-08. This is due to the symmetry of the instances and the large number of simultaneous potential conflicts. As discussed in Section VI-A, the set of possible decisions becomes prohibitively large for these scenarios. Gurobi is then stopped before a feasible solution could be found.

The most surprising consequence of (24)-(26) is that ONE is better than TIME on all three aspects. The main reason for this is that, overall, fewer approximations are needed to build ONE than TIME. Indeed, no approximation is needed in ONE to model the separation constraints or the upper bounds of acceleration and yaw rate. The disadvantage of ONE is the time decomposition needed to get trajectories with more

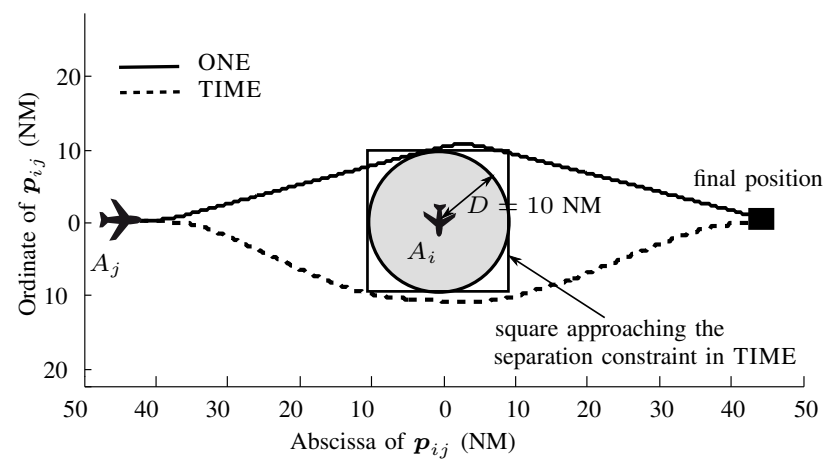

(a) Solutions of ONE and TIME

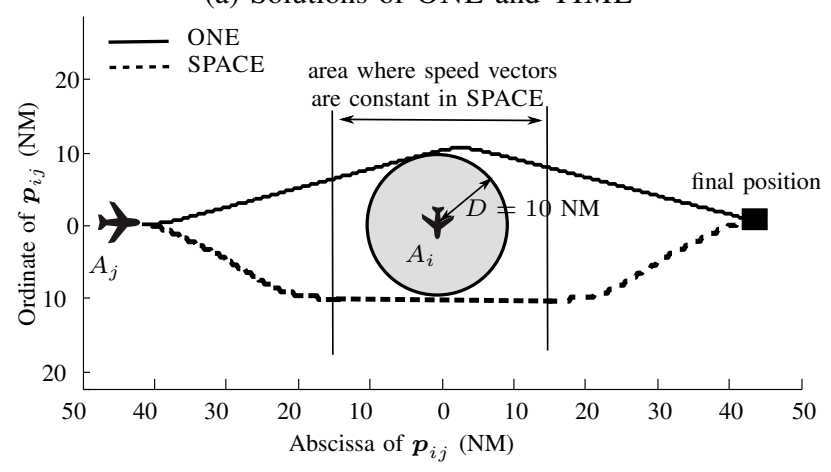

(b) Solutions of ONE and SPACE

Fig. 11: Resolution of a conflict between two aircraft

than one modification of the speed vector. Since the complete solution is the aggregation of the solutions obtained at each time step, there is a necessary loss with regards to optimality. This is compensated for by the fact that maneuvers have to be started as soon as potential conflicts are detected to be efficient. Consequently, the maneuvers computed at the first time step need only a few adjustments during the following iterations.

The comparison between SPACE and the other two MILPs is less straightforward, because SPACE is either the worst or the best on each aspect. For instance, Figure 10b shows that the average cost for SPACE is approximately twice that for 
ONE. Another drawback of SPACE is that $35 \%$ to $40 \%$ of the instances of R07 and R08 had no conflict-free solutions (see Figure 10a). In contrast, Figure 10c highlights that SPACE is solved in a few seconds. This alone could lead to a preference for SPACE over TIME, because ATC has a strong need for reactivity. ONE appears however to be a better compromise on the three aspects that we focused on. Indeed, the only advantage of SPACE is that it is solved faster than ONE, but the latter model is still solved in a small computational time. Moreover, if need be, Algorithm 1 may be stopped as soon as it finds maneuvers that avoid every loss of separation. In most cases, this is the first time step.

From a modeling point of view, SPACE involves tedious mathematical developments and several restrictions on the maneuvers. It also requires that the aircraft follow tracks. This is the case in the current route network, but new technologies may allow for more flexibility. It is difficult to say whether new operational needs could be taken into account in a space-discretized-model without other costly approximations. In contrast, one-maneuver models are relatively simple.

\section{CONCLUSION}

This article is motivated by the difficulty of objectively comparing the models and algorithms developed for the air conflict resolution problem. We study three MILPs representing the diversity of the state of the art for the CR problem restricted to planar and deterministic motion. They are respectively obtained by focusing on a small set of points including those where trajectories intersect (SPACE), by sampling the time horizon (TIME), and by assuming that each aircraft performs only one maneuver (ONE). We describe major revisions of two existing time-discretized and one-maneuver models that are necessary to consider the same constraints and objective function. Every model then minimizes a combination of fuel consumption and delay and avoids losses of separation through speed and heading maneuvers. Furthermore, each aircraft respects dynamical constraints on the velocity, acceleration, and yaw rate, and each reverts to its planned trajectory after the maneuvers.

We then conduct a computational comparison on a benchmark of artificial instances representing very dense traffic. The results reveal that every model may be solved to find conflictfree and economically efficient trajectories in nearly every situation. Our analysis focuses on the number of conflict-free solutions, their average costs, and the computational times. It shows that the apparently simpler one-maneuver model represents the best compromise. The model fails to produce a conflict-free solution on only one of 1600 instances, and the computational time is compatible with an operational implementation.

Future research could carry on the computational comparison that has been started here. The most promising models and algorithms using nonlinear programming, metaheuristics, potential techniques, etc. could be adapted to satisfy the same constraints and minimize the same objective function. Since the instances we used cannot reflect the complexity of real traffic, a more complete comparison could also involve an extended benchmark including instances originating from real traffic data.

It will also be necessary to study how more realistic assumptions could be introduced in the models and how they would impact the performance of the algorithms. To be specific, the wind and the presence of human controllers and pilots in the loop will introduce a shift between the predicted and the observed maneuvers. A consistent approach would be to design robust resolution maneuvers that would account for uncertainties on speed vectors and on the starting time of the maneuvers. For instance, Irvine [32] computes an analytical expression of the probability of conflict for independent and normally distributed uncertainties on aircraft speed. Considering that an error on the starting time of the maneuvers results in lateral and longitudinal shifts in the initial positions of the aircraft, the method of Irvine could be extended to include uncertainties on the starting time of the maneuvers. The expression of the probability of conflict could then be used to compute an increased separation distance that guarantees a low probability of conflict. This would allow to introduce several sources of uncertainties without modifying the structure of the models.

Another impact of uncertainties is that maneuvers should not be started as soon as potential conflicts are detected, since the errors grow with the time to conflict. As a consequence, it would be interesting to study the impact of uncertainties on the optimal starting time of the maneuvers. None of the three MILPs would be compatible with this study without major modifications. Given the simplicity and the performance of ONE, it seems to be a promising choice to deal with this issue. One important step of this work will be to revise the constraints to allow non simultaneous maneuvers.

\section{REFERENCES}

[1] ICAO, "Procedures for air navigation services - Rules for the air and traffic services," Tech. Rep., 1996.

[2] T. Lehouillier, C. Allignol, J. Omer, and F. Soumis, "Interactions between operations and planning in air traffic control," in International Conference on Research in Air Transportation (ICRAT), 2014.

[3] J. K. Kuchar and L. C. Yang, "A review of conflict detection and resolution modeling methods," IEEE Transactions on Intelligent Transportation Systems, vol. 1, no. 4, pp. 179-189, 2000.

[4] F. J. Martín-Campo, "The collision avoidance problem: Methods and algorithms," Ph.D. dissertation, 2010.

[5] N. Durand, "Optimisation de trajectoires pour la résolution de conflits en route.” Ph.D. dissertation, INPT, 1996.

[6] A. Bicchi and L. Pallottino, "On optimal cooperative conflict resolution for air traffic management systems," IEEE Transactions on Intelligent Transportation Systems, vol. 1, no. 4, pp. 221-231, 2000.

[7] A. U. Raghunathan, V. Gopal, D. Subramanian, L. T. Biegler, and T. Samad, "Dynamic optimization strategies for three-dimensional conflict resolution of multiple aircraft," Journal of Guidance, Control, and Dynamics, vol. 27, no. 4, pp. 586-594, 2004.

[8] C. Frese and J. Beyerer, "Planning cooperative motions of cognitive automobiles using tree search algorithms," in KI 2010: Advances in Artificial Intelligence, ser. Lecture Notes in Computer Science, R. Dillmann, J. Beyerer, U. Hanebeck, and T. Schultz, Eds. Springer Berlin/Heidelberg, 2010, vol. 6359, pp. 91-98.

[9] N. Durand, J.-M. Alliot, and J. Noailles, "Automatic aircraft conflict resolution using genetic algorithms," in Symposium on Applied Computing, 1996.

[10] F. Borrelli, D. Subramanian, A. U. Raghunathan, and L. T. Biegler, "MILP and NLP techniques for centralized trajectory planning of multiple unmanned air vehicles," in American Control Conference, vol. 1-12, 2006, Proceedings Paper, pp. 5763-5768. 
[11] J. Omer, "Modèles déterministes et stochastiques pour la résolution numérique du problème de maintien de séparation entre aéronefs," Ph.D. dissertation, Institut Supérieur de l'Aéronautique et de l'Espace (ISAE), 2013.

[12] Gurobi Optimization, Inc., "Gurobi optimizer reference manual," 2012. [Online]. Available: http://www.gurobi.com

[13] T. Schouwenaars, B. DeMoor, E. Feron, and J.How, "Mixed integer programming for multi-vehicle path planning," in European Control Conference, 2001, pp. 2603-2608.

[14] A. Richards and J. How, "Aircraft trajectory planning with collision avoidance using mixed integer linear programming," in American Control Conference, vol. 3, 2002, pp. 1936-1941.

[15] T. Schouwenaars, "Safe trajectory planning of autonomous vehicles," Ph.D. dissertation, Massachusetts Institute of Technology, Department of Aeronautics and Astronautics, Cambridge MA, 2006.

[16] J. Omer and J.-L. Farges, "Hybridization of nonlinear and mixed-integer linear programming for aircraft separation with trajectory recovery," IEEE Transactions on Intelligent Transportation Systems, vol. 14, no. 3 , pp. 1218-1230, 2013.

[17] L. Pallottino, E. Feron, and A. Bicchi, "Conflict resolution problems for air traffic management systems solved with mixed integer programming," IEEE Transactions on Intelligent Transportation Systems, vol. 3, pp. 3-11, 2002.

[18] A. Vela, S. Solak, J. Clarke, W. Singhose, E. Barnes, and E. Johnson, "Near real-time fuel-optimal en route conflict resolution," IEEE Transactions on Intelligent Transportation Systems, vol. 11, pp. 826-837, 2010.

[19] H. Augris, A. Vela, E. Salaun, M. Gariel, and E. Feron, "A conflict resolution algorithm for reduced controller taskload," in AIAA Infotech@Aerospace, 2010.

[20] A. Alonso-Ayuso, L. Escudero, and F. Martìn-Campo, "Collision avoidance in air traffic management: A mixed-integer linear optimization approach," IEEE Transactions on Intelligent Transportation Systems, vol. 12 , no. 1 , pp. 47-57, 2011.

[21] A. Vela, S. Solak, W. Singhose, and J.-P. Clarke, "A mixed integer program for flight-level assignment and speed control for conflict resolution," in Proceedings of the 48th IEEE Conference on Decision and Control, 2009, pp. 5219-5226.

[22] A. Alonso-Ayuso, L. Escudero, P. Olaso, and C. Pizarro, "Conflict avoidance: 0-1 linear models for conflict detection \& resolution," TOP, Springer, pp. 1-20, 2011.

[23] D. Rey, C. Rapine, R. Fondacci, and N.-E. E. Faouzi, "Potential air conflicts minimization through speed regulation," in TRB 91st Annual Meeting Compendium of Papers, 2012.

[24] S. Cafieri and N. Durand, "Aircraft deconfliction with speed regulation: New models from mixed-integer optimization," Journal of Global Optimization, vol. 58, no. 4, pp. 613-629, 2013.

[25] J. Omer, "A space-discretized mixed-integer linear model for air-conflict resolution with speed and heading maneuvers," Computers \& Operations Research, vol. 58, pp. 75-86, 2015.

[26] C. Frese and J. Beyerer, "A comparison of motion planning algorithms for cooperative collision avoidance of multiple cognitive automobiles," in IEEE Intelligent Vehicles Symposium, 2011, Proceedings Paper, pp. 1156-1162.

[27] H. Erzberger, R. A. Paielli, D. R. Isaacson, and M. M. Eshow, "Conflict detection and resolution in the presence of prediction error," in $1^{\text {st }}$ USA/Eur. Air Traffic Management Research Development Seminar, 1997.

[28] G. Chaloulos and J. Lygeros, "Effect of wind correlation on aircraft conflict probability," AIAA Journal of Guidance, Control, and Dynamics, vol. 30, pp. 1742-1752, 2007.

[29] R. A. Paielli, "Modeling maneuver dynamics in air traffic conflict resolution," Journal of Guidance, Control, and Dynamics, vol. 26, pp. 407-415, 2003.

[30] SESAR Joint Undertaking, "European ATM master plan, edition 2," Tech. Rep., 2012.

[31] A. Nuic, "User manual for the Base of Aircraft Data (BADA)," Eurocontrol, Tech. Rep. 11/03/08-08, 2011.

[32] R. Irvine, "A geometrical approach to conflict probability estimation," Air Traffic Control Quarterly, vol. 10, pp. 85 - 113, 2002.

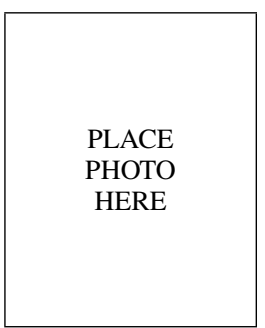

and robotics.
Jérémy Omer was born in Paris, France, in 1982. He received a B.S. degree in electrical engineering from Supelec, France, and an M.S. degree in applied mathematics from Polytechnique Montreal, Canada, in a double-degree program in 2006. He received a Ph.D. in 2013 from ISAE, Toulouse, for research conducted under the guidance of Dr. G. Verfaillie and Dr. T. Chaboud at Onera, the French Aerospace Lab. His research interests include decomposition algorithms for linear programming and optimization techniques applied to air-traffic systems, logistics 